\title{
Time Reversal of electromagnetic waves
}

\author{
G. Lerosey, J. de Rosny, A. Tourin, A. Derode, G. Montaldo, M. Fink \\ Laboratoire Ondes et Acoustique, ESPCI, Université Paris VII, UMR 7587, \\ 10 rue Vauquelin, 75005 Paris, France
}

We report the first experimental demonstration of time-reversal focusing with electromagnetic waves. An antenna transmits a 1- $\mu$ s electromagnetic pulse at a central frequency of $2.45 \mathrm{GHz}$ in a high-Q cavity. An other antenna records the strongly reverberated signal. The time-reversed wave is built and transmitted back by the same antenna acting now as a time-reversal mirror. The wave is found to converge to its initial source and is compressed in time. The quality of focusing is determined by the frequency bandwidth and the spectral correlations of the field within the cavity.

In acoustics, time reversal experiments can be carried out with broadband wave forms [1]. In such experiments, a source sends a short pulse that propagates through a more or less complex (but ideally non dissipative) medium and is captured by a transducer array, termed a Time Reversal Mirror (TRM). The recorded signals are digitized, stored in electronic memories, time-reversed, re-analogized and finally transmitted back by the TRM. The timereversed wave is found to converge back to its source all the more accurately than the medium is complex and the frequency bandwidth is larger [2]. This is very appealing for applications in telecommunications. Indeed, it was recently shown with ultrasonic waves that it is possible to take advantage of the complexity of a medium to convey more information through it by means of a TRM [3]. From a practical point of view, a TRM was used to focus random series of bits simultaneously to different receivers which were only a few wavelengths apart. In the language of communication, it corresponds to a MIMO-MU configuration (Multiple Input, Multiple Output Multiple Users). While the transmission was free of error when strong multiple scattering occured in the propagation medium, the error rate was huge in the homogeneous medium (free space) due to crosstalk between receivers. Indeed, the spatial resolution of a TRM can be much thinner in a multiple scattering medium than in free space. It is the well-known "super-resolution" that has been experimentally highlighted [2,4] and theoretically discussed [2,5] in the past.

Is it possible to transpose this idea to the electromagnetic case? It is a challenging question because in many real environments (buildings or cities), microwaves with wavelengths between 10 and $30 \mathrm{~cm}$ are scattered by objects such as walls, desks, cars, etc which produces a multitude of paths from the transmitter to the receiver. In such situations, a timereversal antenna should be able not only to compensate for these multipaths, but also increase the information transfer rate thanks to the many reflections/reverberations, as it was already shown with ultrasound [3]. However, the first step is to prove the feasability of a time reversal experiment with electromagnetic waves in the $\mathrm{GHz}$ range. It is the aim of this letter. To that goal, we present the first onechannel electromagnetic time reversal mirror (TRM) working around $2.45 \mathrm{GHz}$.

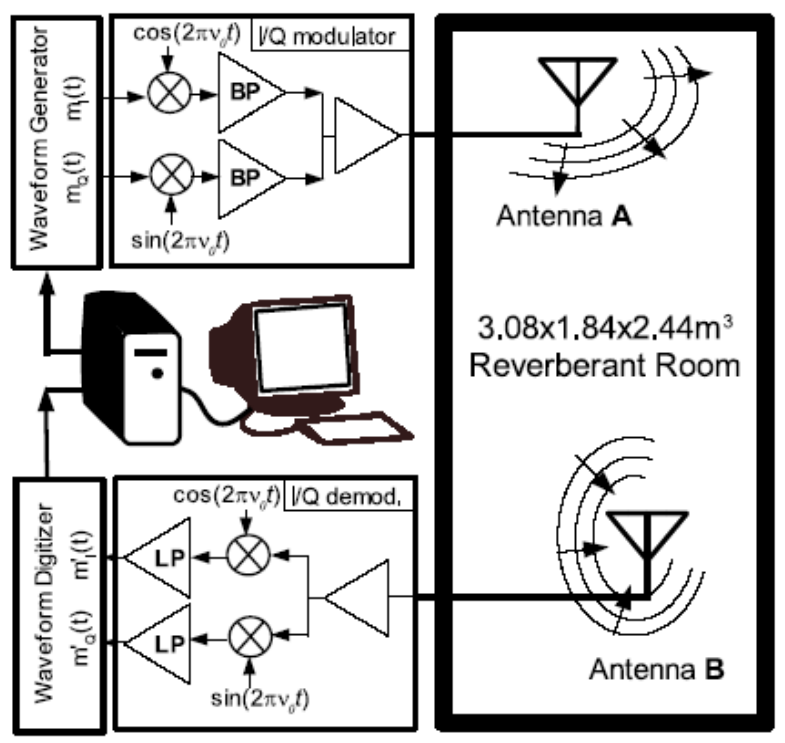

Fig. 1 Sketch of a transmit/receive experiment. The baseband signal $m_{I}(t)$ (resp. $\left.m_{Q}(t)\right)$ is fed into a mixer that multiplies it by $\cos \left(2 \pi \mathrm{f}_{0} t\right)\left(\right.$ resp. $\left.\sin \left(2 \pi \mathrm{f}_{0} t\right)\right)$, with $f_{0}=2.45 \mathrm{GHz}$. After amplification, the output $\mathrm{rf}$ signal is sent by antenna $A$, which is located in the reverberating room. The reverberated field is recorded by antenna $B$. Its spectrum is downshifted to the baseband by a multiplier and a low-pass filter. The resulting signals $m_{I}^{\prime}(t)$ and $m^{\prime}{ }_{Q}(t)$ are sampled and stored in the computer memory. Then they can be timereversed, re-analogized and, following the same principle, sent back by antenna $B$ while antenna $A$ acts now as a receiver.

From a practical point of view, the main difficulty to transpose the time reversal technique developed for ultrasound directly to the electromagnetic case lies in the much higher sampling 
frequencies that are needed to digitize radiofrequency (RF) signals. One way to overcome this limitation is to work only with quasimonochromatic signals and to do a phase conjugation using the so-called three-wave or four-wave mixing in a non linear material in order to naturally produce the analogic phase-conjugated wave [6]. Here, we want to perform a truly broadband time reversal for an electromagnetic pulse $m(t) \cos \left(2 \pi f_{0} t+\varphi\right)$, with $f_{0}$ the carrier frequency and $m(t)$ a lower frequency signal ("baseband signal"). The timereversal operation can be decomposed in two steps: phase conjugation of the wave carrier $f_{0}$, plus timereversal of the baseband signal $m(t)$. The advantage is that the sampling of the baseband signal requires a sampling frequency much lower than $2 f_{0}$.

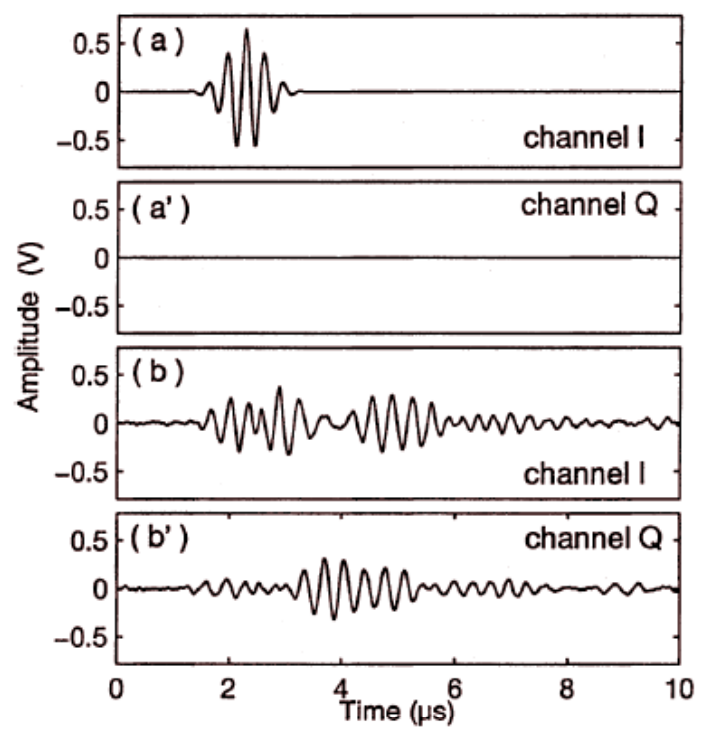

Fi

Fig. 2. (a) and (a'): baseband representation (resp. $m_{I}(t)$ and $m_{Q}(t)$ ) of the signal transmitted by antenna $A$. (b) and (b'): baseband representation (i.e. $m_{I}(t)$ and $m_{Q}{ }_{Q}(t)$ ) of the signal reverberated inside the cavity and received by antenna $B$.

The experiment takes place in a strongly reverberant cavity with dimensions $3.08 \mathrm{~m} \times 1.84 \mathrm{~m} \times$ $2.44 \mathrm{~m}$. Using an arbitrary waveform generator, we deliver a short pulse $m_{I}(t)$ (central frequency $3 \mathrm{MHz}$, $6 \mathrm{~dB}$ bandwith $2 \mathrm{MHz}$ ) to the $I$ analog input (cf. Fig. 2.(a)) of the transmit board. No signal is delivered to the $Q$ analog input (Fig. 2.(a')). A mixer upconverts this signal to the $\mathrm{GHz}$ band and delivers $e(t)=m_{I}(t) \cos \left(2 \pi f_{0} t\right)$. Then the waveform $e(t)$ is transmitted by antenna $A$. After propagation, the signal $s(t)=m_{I}^{\prime}(t) \cos \left(2 \pi f_{0} t\right)+m^{\prime}{ }_{Q}(t) \sin \left(2 \pi f_{0} t\right)$ is recorded by antenna $B$ and downconverted to produce the $I$ and $Q$ components of the output signal $m_{I}^{\prime}(t)$ and $m_{Q}^{\prime}(t)$ that can be observed at the oscilloscope ( Fig. 2. (b) and (b’) ). The received signals last more than $8 \mu$ s, i.e. eight times longer than the initial baseband pulse, or 3200 periods of the RF wave. The RF wave has travelled more than two kms in the $14-\mathrm{m}^{3}$ cavity, i.e. undergone $\sim 6000$ reflections.
Next, our goal is to time-reverse the received radio signal $s(t)$. To that end, the baseband $I$ and $Q$ signals $m^{\prime}{ }_{I}$ and $m^{\prime}{ }_{Q}$ are digitized by the oscilloscope at a $40-\mathrm{MHz}$ sampling rate, sent to a computer and timereversed. The wave carrier has to be conjugated too. The following step consists in re-analogizing the timereversed $I$ and $Q$ signals, and encoding them on the phase-conjugated wave carrier: the resulting RF signal writes $m_{I}^{\prime}(-t) \cos \left(2 \pi f_{0} t\right)-m_{Q}{ }_{Q}(-t) \sin \left(2 \pi f_{0} t\right)=s(-t)$. It is then transmitted back by antenna $B$. After propagation, the RF signal received on antenna $A$ is downconverted to baseband. As can be seen in Fig. 3.(a), the received signal on channel $I$ is compressed in time and recovers its initial duration.

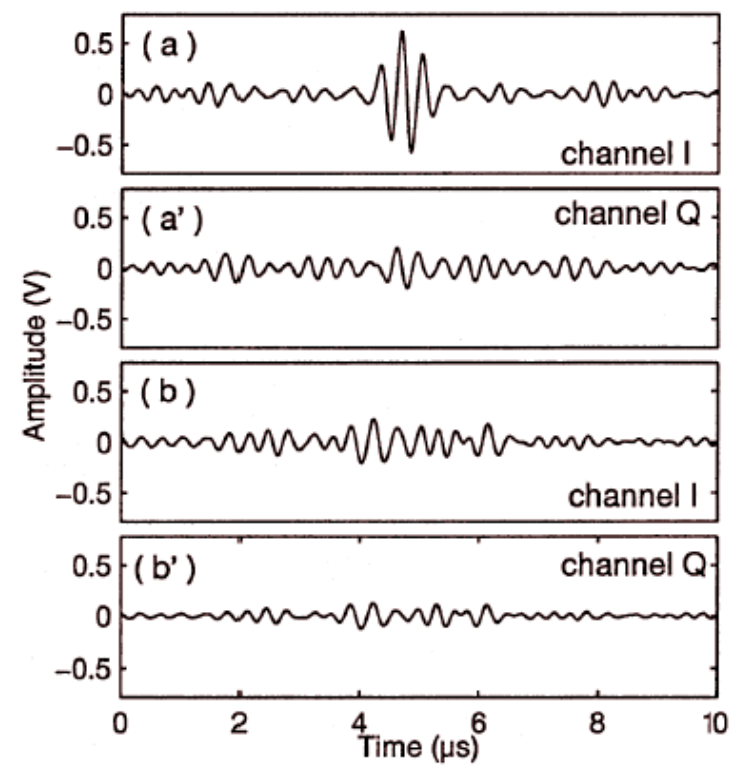

Fig. 3. (a) and (a') : baseband representation of the signal received by antenna $A$ after time-reversal. (b) and (b'): baseband representation of the signal received several wavelengths away from antenna $A$ after time-reversal.

Actually, since the reverberated wavefield has been captured by a single antenna, the time-reversal operation is not perfect. The waveform that is recreated is not the exact replica of the initial pulse: there are sidelobes around the peak on channel $I$, and a signal is measured on channel $Q$ (Fig. 3. (a') ) although nothing was sent on that channel. Similar effects have been observed for time-reversal of ultrasonic waves: it can be shown that the peak-to-noise ratio in a one-channel time-reversal experiments varies as $\sqrt{\Delta f / \delta f}$ where $\Delta f$ is the available bandwidth and $\delta f$ defines the correlation frequency of the reverberated field [2]. $\delta f$ is the characteristic width of the field-field correlation function $\int\left\langle\psi(f) \psi^{*}(f+\delta f)\right\rangle d f$, with $\psi$ the scattered electromagnetic field. Therefore one could expect an even stronger pulse compression if the bandwidth was larger, or the correlation frequency smaller. Given the dimensions of the cavity, the Heisenberg time (i.e. the 
inverse of the mean distance between eigenmodes) is $\mathrm{t}_{\mathrm{H}} \sim 80 \mu \mathrm{s}$. But the characteristic absorption time is $t_{a}=$ $3.6 \mu \mathrm{s}$, therefore the modes are not resolved and the correlation frequency $\delta f$ is determined by $t_{a}$ rather than $\mathrm{t}_{\mathrm{H}}$. Taking $\Delta f=2 \mathrm{MHz}$ and $\delta f=1 / t_{a} \approx 280 \mathrm{kHz}$ leads to a predicted peak-to-noise ratio roughly equal to 3 , comparable to our experimental results.

The experiment shows that time reversal is able to compensate for multiple reverberation and recreate a short electromagnetic pulse at the source. But there is more to it: we have also verified that the amplitude of the re-created signal is stronger at antenna $A$ than anywhere else in the cavity ( Fig. 3. (b) and (b') ), i.e. the time reversed wave is spatially focused. This is consistent with past experiments using ultrasound in reverberant or strongly scattering media that proved that even with a one-channel time-reversal device, the pulse is sharply focused at the source and only at the source, the noise level surrounding the source being, once again, controlled by $\sqrt{\Delta f / \delta f}$ [2]. Here, the role of reverberation as well as the large frequency band must be emphasized. Since we only have a one-channel time-reversal, no focusing would occur in free space no matter how large the bandwidth; in a reverberating medium, no focusing would occur either if the bandwitdh was too narrow, as is the case in classical phase-conjugation techniques. Indeed, imagine a single time-reversal antenna trying to focus a pure monochromatic wave on some receiver; the phase-conjugated wave has no reason at all to be focused on this point since the antenna only sends back a sinusoidal spherical wave through the medium. But if the frequency bandwidth $\Delta f$ is much larger than the correlation frequency $\delta f$, then the spectral components of the reverberated field $\psi$ at two frequencies apart by more than $\delta f$ are decorrelated: there are roughly $\Delta f / \delta f$ decorrelated components in the spectrum of the reverberated signals. When we time-reverse (i.e. phase-conjugate coherently all along the bandwidth, and not just at one frequency) all these components, they add up in phase at $t=0$ at the receiver position, because all the phases have been set back to 0 all along the bandwidth. Thus, the amplitude at this position and at this time increases as $\Delta f / \delta f$ whereas outside the receiver position (and outside $t=0$ ), the various frequency components add up incoherently and their sum rises as $\sqrt{\Delta f / \delta f}$. On the whole, the peak-tonoise ratio increases as $\sqrt{\Delta f / \delta f}$ as the bandwidth is enlarged. It explains why a time-reversal experiment is possible even with one single TR channel [2,5,7], as long as the frequency bandwidth is sufficiently larger than the correlation frequency of the propagation medium.
We have presented the first time reversal experiment for electromagnetic waves in the $\mathrm{GHz}$ domain. The experiments were carried out in a reverberating cavity. To avoid digitizing the radio signals at $\mathrm{GHz}$ frequencies, we have time reversed the baseband signals and phase-conjugated the wave carrier. Thanks to reverberation, the resulting signal at the source is compressed in time and in space with a signal-to-noise ratio depending on the ratio of the bandwidth to the frequency correlation of the medium. In the future, a larger bandwidth has to be used to improve both spatial and temporal focusing. This seems to be possible with the emergence of ultra wide band electromagnetics components. Thus time-reversal focusing could have promising applications in the field of wide-band wireless communications in complex reverberant environments.

\section{ACKNOWLEDGMENTS}

The authors wish to acknowledge the "Département de Recherche en Electromagnétisme, Laboratoire Signaux et Systèmes, Supélec”, Gif-surYvette, France (www.lss.supelec.fr) and particularly A. Azoulay and V. Monebhurrun for having let us use their reverberant cavity, as well as the "Conseil Régional d'Ile de France" and the "Conseil Départemental de l'Essonne” who financed the cavity. This work is a part of the research projects developed within the "Groupement de Recherche" ImCoDe (GDR 2253, CNRS, http://lpm2c.grenoble.cnrs.fr/IMCODE/IMCODE.html ) .

\section{REFERENCES}

1. M. Fink, Physics Today 50,34 (1997).

2. A. Derode, A. Tourin and M. Fink, Phys. Rev. E 64, 036606 (2001).

3. A. Derode, A. Tourin, J. de Rosny, M. Tanter, S. Yon, M. Fink, Phys. Rev. Lett. 90, 014301-1 (2003).

4. A. Derode, P. Roux, M. Fink, Phys. Rev. Lett. 75, 4206 (1995).

5. P. Blomgren, G. Papanicolaou, H. Zhao, J. Acoust. Soc. Am., 111, 203 (2002).

6. D.M. Pepper, Opt. Eng., 21, 156 (1982). Yian Chang, H. R. Fetterman, I.L. Newberg, and S.K. Panaretos, Appl. Phys. Lett. 72, 745 (1998).

7. C. Draeger, M. Fink, Phys. Rev. Lett. 79, 407 (1997). 